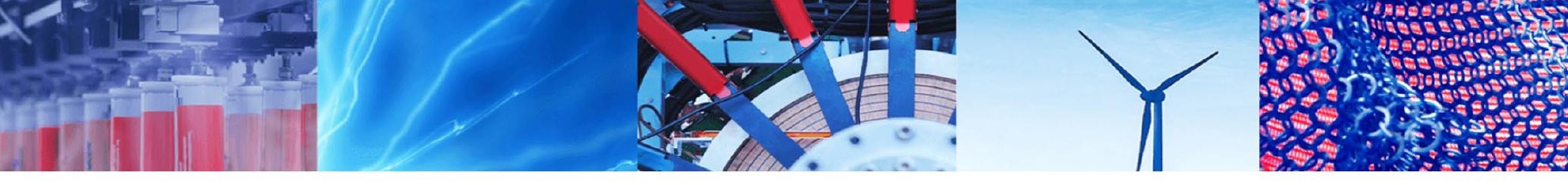

Research Article

\title{
Sound absorption performance of needle-punched nonwovens and their composites with perforated rubber
}

\author{
Chureerat Prahsarn $^{1}$ (D) $\cdot$ Wattana Klinsukhon $^{1} \cdot$ Natthaphop Suwannamek $^{1} \cdot$ Prapudsorn Wannid $^{1} \cdot$ Sirada Padee $^{1}$
}

Received: 24 January 2020 / Accepted: 2 March 2020 / Published online: 5 March 2020

(c) Springer Nature Switzerland AG 2020

\begin{abstract}
Sound absorption performance of needle-punched nonwovens containing polyester fibers of different sizes ( 7 and 15 denier) and configurations (hollow, 4-hole, hollow conjugated, and hollow trilobal), as well as their composites with perforated rubber layer were investigated. From results, nonwovens of finer 7-denier fibers exhibited higher sound absorption coefficient (a) than that of coarser 15-denier fibers. This was due to greater amount of fibers in nonwovens and larger fiber surface area, thus longer tortuous path for sound waves to travel. Nonwoven of 4-hole fibers (7-4H) showed higher sound absorption performance than that of single hole fibers $(7-\mathrm{H})$ due to increase in surface areas of small holes inside the fibers, thus increasing tortuous path. Likewise, increase in hollow area in the hollow conjugated fibers (15-HC), and angular configuration in the hollow triangular fibers $(15-\mathrm{HT})$ resulted in greater sound absorption coefficient, compared to those of hollow fiber counterpart (15-H). Increasing nonwoven layer to 2 and 3 layers yielded an increase in sound absorption coefficient due to greater thickness and more air gaps between the nonwoven layer. The rubber/nonwoven composites, where perforated rubber layer was inserted between nonwoven layers, exhibited higher sound absorption coefficient in a low-frequency range. This study demonstrated that sound absorption performance could be enhanced through a wide frequency range by employing nonwoven of fine fibers having irregular shapes and large hole area, in combination with perforated elastic rubber.
\end{abstract}

Keywords Sound absorption · Nonwovens · Fiber sizes · Fiber configurations · Perforated rubber

\section{Introduction}

Undesirable and high-level noise is considered a potential threat to human comfort and health [1-3]. A variety of acoustic materials has been used as either sound barriers or sound absorbers to reduce such noise or sound to a comfort level or silence. Solid and impervious materials, for examples, act as a sound barrier, which reflects the incoming sound in order to prevent sound transmission. On the contrary, porous materials, including foams and fibrous materials with internal pores, act as sound absorber, especially in a high frequency range [1, 2]. Nonwovens are fibrous materials, which are assembled directly from fibers. Their porous structures and high surface areas make nonwovens attractive for being used as sound absorbers for many technical applications, for instance in building and automotive insulations. Porosity in the structures, large fibers surface area, as well as fibers interlocking are frictional elements that provide resistance to sound wave propagation. When the sound wave enters into nonwoven, it moves through tortuous passages and contacts with the fiber surface, resulting in energy dissipation into heat loss, i.e. loss of sound energy [3-5]. Although materials with porous structure exhibit good acoustic absorption properties in a high frequency range, their performance at a low frequency range was

Chureerat Prahsarn, chureerp@mtec.or.th | ${ }^{1}$ National Metal and Materials Technology Center, 114 Thailand Science Park, Paholyothin Rd., Klong 1, Khlong Luang, Pathumthani 12120, Thailand. 
poor [6]. To improve sound absorption at low frequency, an addition of viscous interlayer such as soundproofing material could be used to increase the damping effect. The acoustic waves from the incident sound cause shear strain within the viscous interlayer material, and then are transformed into heat energy as a result of the high damping characteristics of interlayer material.

Sound absorption characteristics of nonwoven materials have been studied in many aspects [2-9]. Senggupta [2] studied effects of different parameters such as fabric type, number of layers, area density and fiber type on sound reduction of needle-punched nonwovens. It was concluded that nonwovens exhibited higher sound reduction than woven fabrics, and nonwoven of Jutepolypropylene (1:1) blend showed the maximum sound reduction compared to Jute, polypropylene, polyester and other jute-polypropylene blends (3:1 and 1:3). Increasing number of nonwoven layers increased sound reduction, before reaching a plateau of the maximum point. Tascan and Vaughn [4] studied the effects of total surface area and fabric density on sound absorption performance of needle-punched nonwovens. They reported better sound absorption in nonwovens of finer fibers with various crosssectional shape such as trilobal and 4DGs, compared to that of coarser fibers with round shape. Similar observation was reported by Abdelfattah et al. [7] that nonwovens containing high percent of hollow polyester fibers exhibited higher sound absorption, compared to that of typical polyester fibers. This was explained due to hollow structure of the fibers, which contain air inside, thus increasing ability of nonwoven to absorb sound waves. Lee and Joo [8] investigated sound absorption coefficient of recycled polyester nonwovens with different fiber contents and fineness. The nonwovens were also combined with other sound reflecting materials such as PP film, aluminum foil, and spunbond nonwoven for further investigation. They concluded that nonwovens with higher proportion of fine fibers could dissipate more sound due to more contact area to sound wave and thus high resistance through vibration of the air. Insertion of resonant panel contributed to increase in sound absorption properties of all frequency regions.

Some other studies had also been conducted, using micro-perforated sound absorber, to improve sound absorption properties in a low-medium frequency range, [1, 10-13]. Liu et al. [10] investigated sound absorption capability of multi-layer micro-perforated panel absorber. In their experiments, 3D-printed micro-perforated panels with different perforation ratios were attached to either porous material or air gap. Results showed significant improvement of sound absorption at low to mid frequency, and thus wider broadband sound absorption was obtained, using the designed multi-layer sound absorbers.
Unlike sound absorptive materials, sound barrier materials reduce sound via blocking or soundproof the area. Rubber is highly elastic and temporarily deformed under pressure and loading, thus can be used as a sound barrier. Recent studies on recycled rubber particles from tire known as crumb rubber showed that it could be employed as a filler for noise absorption, especially at a low frequency range [14, 15]. Lou et al. [16] investigated sound absorption properties of perforated flexible-foam/nonwoven composites, having different percent of perforation (5-20\%). The nonwoven laminates made of low melting point polyester (LMPET), flame retardant polyester (FRPET) and recycled Kevlar fibers were fabricated, and PU foam was sandwiched between them to make the composites with porous and resonance structures. From results, the perforated flexible foam/nonwoven composite with 4-mm hole exhibited the highest sound absorption coefficient. With increasing percent of perforation, absorption peak moved towards higher frequency. It was shown that, by combining the nonwoven laminate, increase in sound absorption performance of perforated flexible foam could be achieved over broad frequency range.

In this work, the sound absorption characteristics of polyester nonwovens as well as their composites with perforated rubber had been investigated comprehensively and systematically. The parameters including fiber sizes (7 and 15 denier) and configurations (hollow, 4-hole, hollow conjugated, and hollow trilobal) as well as number of nonwoven layers (1, 2 and 3 ) had been studied to understand their influences on sound absorption coefficients of the obtained nonwovens. Furthermore, a combination of selected nonwoven with perforated elastic material was investigated to achieve higher sound absorption properties, especially in a low-frequency range. In a series of experiments, a rubber layer with different percentages of perforation (0, 3 and $11 \%$ ) was inserted between the nonwoven layers, and sound absorption properties of the nonwoven-rubber composites were determined.

\section{Experimental}

\subsection{Materials}

Polyester staple fibers (64 mm. in length) with different denier (7D, 15D) and configurations were used. For 7-denier, fibers having 1 hole and 4 holes (labelled as $7-\mathrm{H}$ and $7-4 \mathrm{H}$ ) were used, whereas for 15 -denier, 1 -hole, hollow conjugated, and hollow trilobal fibers (labelled as $15-\mathrm{H}$, $15-\mathrm{HC}$, and $15-\mathrm{HT}$, respectively) were used. Their crosssectional features and dimensions were shown in Fig. 1 and Table 1. The $1 \mathrm{~mm}$-thick rubber layers were prepared by casting $20 \mathrm{ml}$. of natural rubber latex (60\% solid) on the 

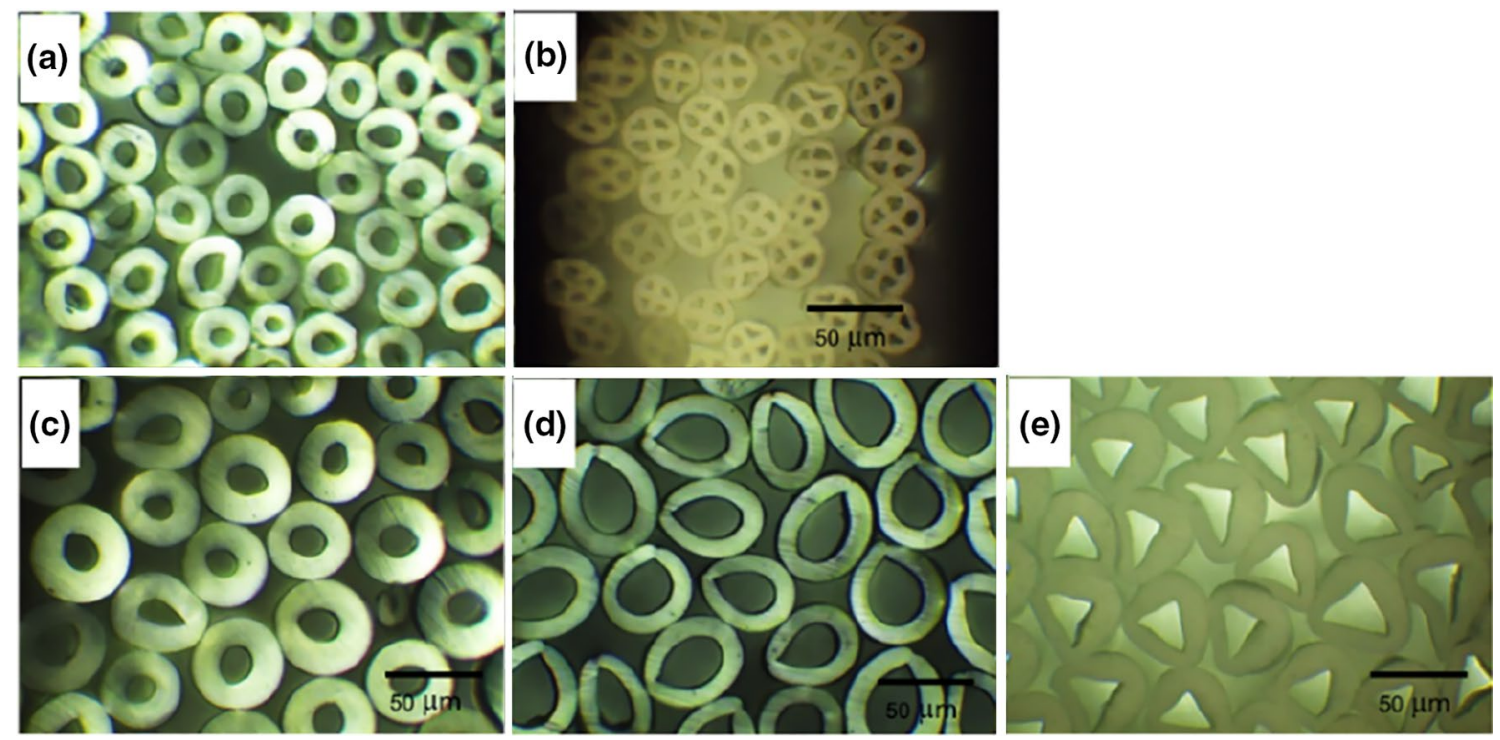

Fig. $1 \mathrm{OM}$ images of PET fibers of different sizes and configurations. a 7-H, b 7-4H, c 15- H, d 15-HC and e 15-HT

Table 1 Dimensions of polyester fibers and properties of their nonwovens

\begin{tabular}{lccrrr}
\hline Fiber & \multicolumn{1}{l}{ 7-H } & \multicolumn{1}{l}{ 7-4H } & \multicolumn{1}{c}{$15-\mathrm{H}$} & \multicolumn{1}{c}{$15 \mathrm{HC}$} & \multicolumn{1}{c}{$15-\mathrm{HT}$} \\
\hline Diameter $(\mu \mathrm{m})$ & $31.68 \pm 1.11$ & $29.66 \pm 1.32$ & $47.30 \pm 2.14$ & $48.73 \pm 2.17$ & $42.85 \pm 2.81^{\mathrm{a}}$ \\
Perimeter $(\mu \mathrm{m})$ & $99.52 \pm 3.49$ & $93.21 \pm 4.16$ & $148.59 \pm 6.74$ & $156.00 \pm 7.09$ & $152.30 \pm 7.53$ \\
\% Hole area & $13.62 \pm 2.38$ & $3.67 \pm 0.96^{\mathrm{b}}$ & $15.28 \pm 1.60$ & $36.50 \pm 2.77$ & $19.11 \pm 2.74$ \\
Nonwovens & & & & & \\
Weight $(\mathrm{gsm})$ & $881.50 \pm 48.74$ & $822.94 \pm 84.71$ & $894.66 \pm 60.67$ & $885.32 \pm 11.48$ & $788.56 \pm 29.98$ \\
Thickness $(\mathrm{mm})$ & $23.03 \pm 0.30$ & $17.50 \pm 1.01$ & $21.23 \pm 2.21$ & $18.30 \pm 1.05$ & $21.34 \pm 0.22$ \\
\hline
\end{tabular}

${ }^{\mathrm{a}}$ Height

${ }^{\mathrm{b}}$ Area of 1 hole

$15 \times 15 \times 0.2 \mathrm{~cm}$. glass mold and allowed to slowly set at ambient temperature for 2 days. The rubber layer (labelled as R), as well as perforated rubber layer with holes of 3-mm diameter, and distance of either 8 or $16 \mathrm{~mm}$ between holes (labelled as $\mathrm{RH} 8$ and $\mathrm{RH} 16$, respectively) were employed for nonwoven-rubber composite (Fig. 2). The measured percent of perforation for $\mathrm{RH} 8$ and $\mathrm{RH} 16$ were $3 \%$, and $11 \%$, respectively.

\subsection{Nonwoven fabrication}

Nonwovens of polyester fibers, having different deniers and configurations, were prepared as the followings. Two hundred grams of fibers were firstly fed into carding machine to form a $120-\mathrm{cm}$ wide fiber web. The fiber webs were then half-folded into $60-\mathrm{cm}$ wide and needlepunched, using needle punching machine, at stroke frequency of 100 time/min and belt speed of $1.5 \mathrm{~m} / \mathrm{min}$, to form nominal $2-\mathrm{cm}$ thick nonwovens of $800 \mathrm{~g} / \mathrm{m}^{2}$ with

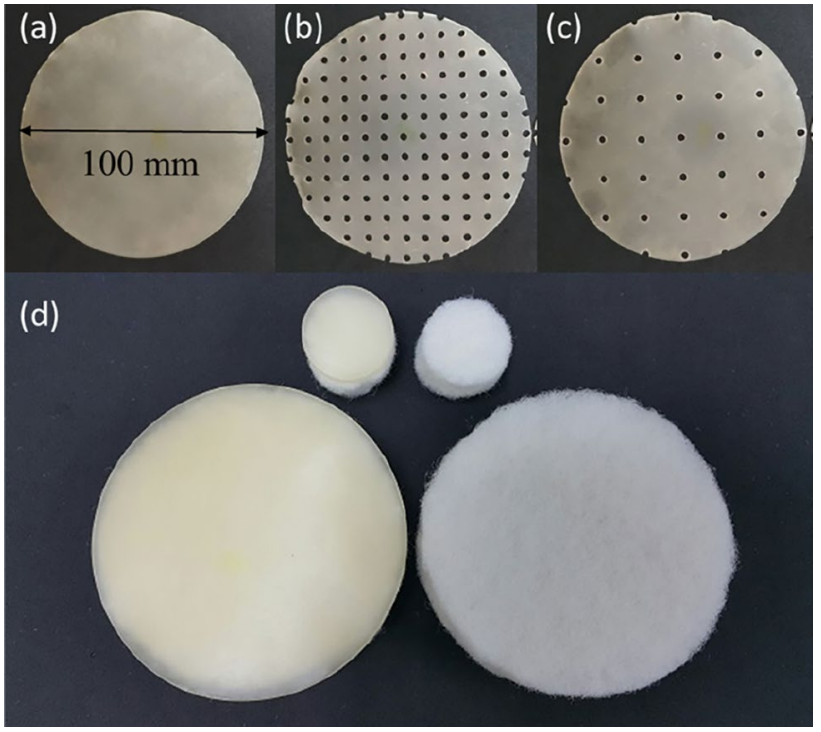

Fig. 2 Rubber layer with different perforation. a R, b RH8, c RH16 and $\mathbf{d}$ an example of nonwoven/rubber composite assembly $(15-\mathrm{H} / \mathrm{R} / 15-\mathrm{H})$ 
punch density of 18 punches $/ \mathrm{cm}^{2}$. Their weights in gsm (g/ $\mathrm{m}^{2}$ ) and thickness were measured and reported in Table 1 .

\subsection{Evaluation on sound absorption properties of nonwovens}

Sound absorption coefficient (a) of nonwoven samples were measured over a frequency range of $100-6400 \mathrm{~Hz}$. (ISO 10534-2), using impedance tube system (BSWA Technology Co., Ltd.). In sample preparation, nonwoven were cut into $100 \mathrm{~mm}$ and $29 \mathrm{~mm}$ diameters for measurements in low and high frequency ranges, respectively. Nonwovens of 1,2, and 3 layers were measured, and their sound absorption coefficients were reported as a function of frequency. For investigation on nonwoven-rubber composite, the $29-\mathrm{mm}$ or $100-\mathrm{mm}$ rubber layer was inserted between the nonwoven layers (Fig. 2) and their sound absorption coefficient were measured.

\section{Results and discussion}

\subsection{Effect of number of nonwoven layer}

Nonwoven samples, in configurations of 1, 2, and 3 layers, were measured for sound absorption properties. Overall, increasing number of nonwoven layer, i.e. increasing thickness, resulted in higher sound absorption coefficients. This observation was in agreement with previous study reported by Sengupta [2] that sound absorption performance increased with increasing nonwoven layers. In Fig. 3, a plot of sound absorption coefficient at different frequencies, measured in nonwovens of 7-4H fibers, was given as an example. For 1 layer, absorption coefficient gradually increased with increasing frequency and reached the maximum plateau $\left(a_{\max }=0.84\right)$ at frequency

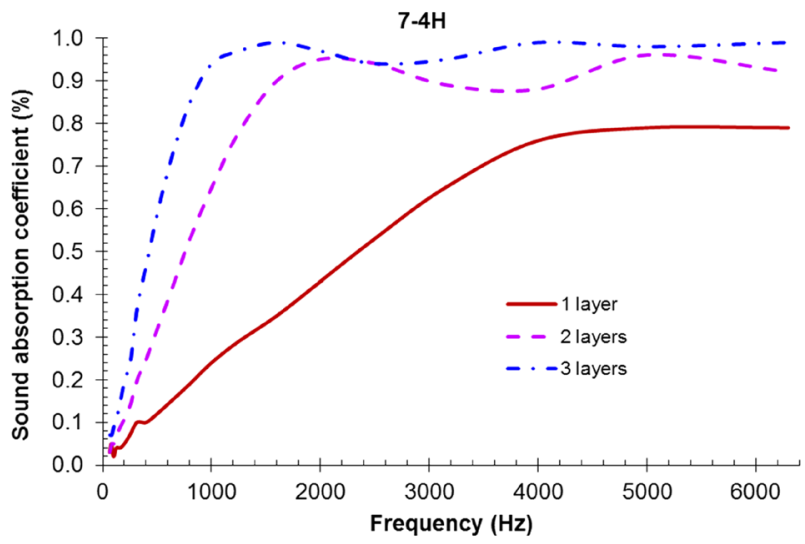

Fig. 3 Sound absorption coefficients of nonwovens with 1, 2 and 3 layers (nonwoven of 7-4H fibers as for example) about $4000 \mathrm{~Hz}$. For 2- and 3-layer configurations, the absorption coefficients increased sharply and reached maximum values of 0.96 and 0.99 at frequencies about 2000 and $1600 \mathrm{~Hz}$, respectively. Similar trends were observed in other nonwoven samples. It was noticed that sound absorption coefficients of 2- and 3-layer nonwoven samples were slightly dropped at some frequency range before increasing again, giving wavy curves. Such characteristic observation could be contributed to the air gaps between the nonwoven layers.

\subsection{Effect of fiber size}

The measured sound absorption coefficients of nonwoven samples, containing different fiber sizes ( 7 and 15 denier), were shown in Fig. 4a-c. In 1-layer configuration (Fig. 4a), nonwovens of finer 7-denier fibers $(7-\mathrm{H}$ and $7-4 \mathrm{H})$ possessed higher sound absorption coefficients, compared to those of coarser 15 -denier fibers $(15-\mathrm{H}, 15 \mathrm{H}-\mathrm{C}$ and $15-\mathrm{HT})$ such that their maximum absorption coefficients were in a range of $0.79-0.86$ versus $0.57-0.72$, respectively. The influence of fiber size was clearly observed when compared between nonwovens of the same configuration; 7-H versus 15-H fibers, whose maximum absorption coefficients were 0.79 and 0.57 , respectively. The higher sound absorption coefficient could be explained by three properties of the fine fibers: (1) larger amount of fine fibers, compared to the coarser fibers in nonwoven of the same weight, resulting in a longer tortuous path in the fibrous structure. (2) Larger surface area of the finer fibers enabled more chance to interact with the sound waves, resulting in higher airflow resistance caused by frictional viscosity through the vibration of the air. (3) Finer fibers could vibrate more easily than the coarser one, leading to conversion of acoustic energy to heat loss. Similar trends in effects of fiber size were also observed in nonwovens of 2- and 3-layer configurations (Fig. 4b, c).

\subsection{Effect of fiber configurations}

Effects of fibers configurations and cross-sectional features on sound absorption characteristics of the nonwovens were also investigated. As shown in Fig. 4a-c, sound absorption coefficient of nonwoven containing 7-denier fibers with four holes $(7-4 \mathrm{H})$ was higher than that of nonwoven containing fibers with single hole $(7-\mathrm{H})$. For 1-layer nonwoven (Fig. 4a), the maximum absorption coefficients of 7- $\mathrm{H}$ and $7-4 \mathrm{H}$ nonwovens were 0.79 and 0.87 , respectively. Both $7-4 \mathrm{H}$ and $7-\mathrm{H}$ fibers had comparable diameters of $29.66 \pm 1.32$ and $31.68 \pm 1.11$ microns, respectively (Table1). Higher sound absorption coefficient observed in nonwoven of 7-4H fibers was partly due to the increase in surface areas of small holes inside the fibers, 


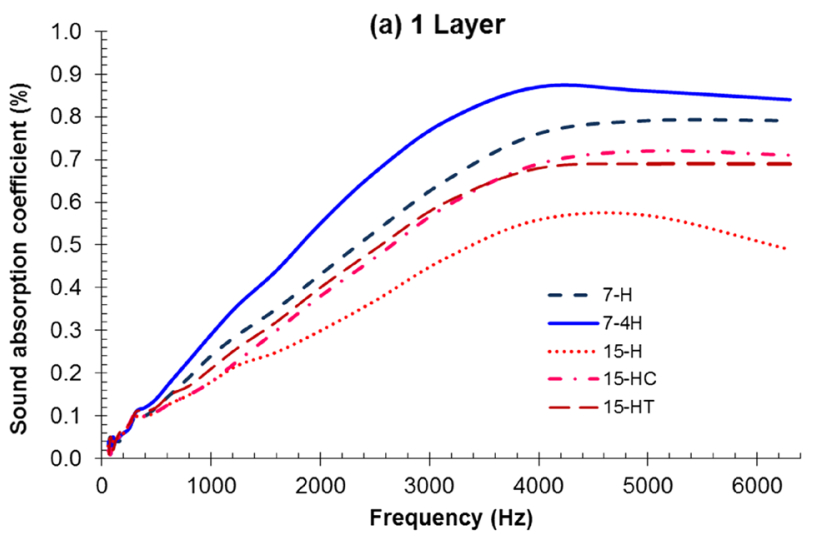

(b) 2 Layers

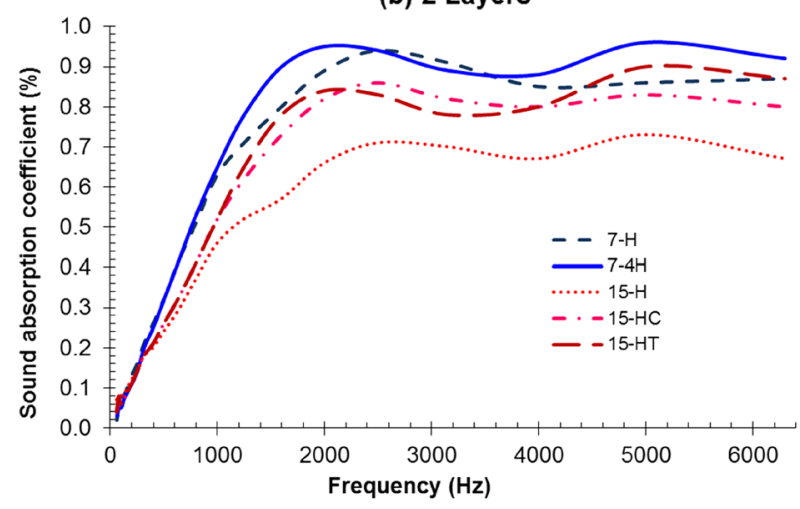

(c) 3 Layers

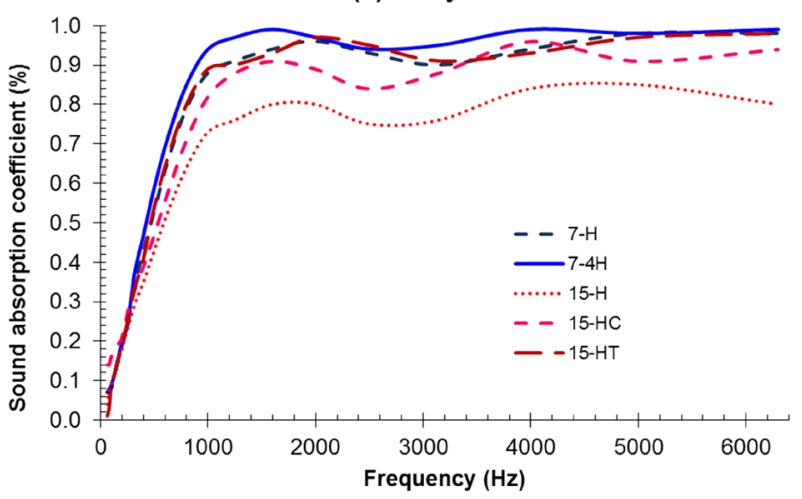

Fig. 4 Sound absorption coefficients of nonwovens with different polyester fibers. a 1 layer, $\mathbf{b} 2$ layers and $\mathbf{c} 3$ layers

thus increasing tortuous path for sound waves. Likewise, higher sound absorption coefficient was observed in nonwoven of thinner wall 15-HC fibers, compared to that of the conventional hollow 15 - $\mathrm{H}$ fibers ( $a_{\max }=0.72$ vs. 0.57 ). Considering their comparable diameters, $48.73 \pm 2.17$ and $47.30 \pm 2.14$ microns for $15-\mathrm{HC}$ and $15-\mathrm{H}$, respectively, larger volume of hollow space in 15-HC fibers (hole area of 36.50 vs. $15.28 \%$ ) could also play a role on sound absorption. The greater volume of air inside holes retarded sound propagation through the nonwoven, resulting in higher absorption coefficient. Similar observation was reported by Abdelfattah et al. that increasing fiber hollow percentage, i.e. air volume, resulted in higher sound absorption efficiency [5]. As shown in Fig. 4, nonwoven containing 15-HT fibers exhibited comparable sound absorption performance, compared to that of $15-\mathrm{HC}$ fibers despite its lower percent of hole area (19.11 vs. $36.5 \%)$. This implied that its triangular shape might also play a role in increased sound absorption coefficient such that its angular configurations scattered the sound in different directions, leading to higher loss of sound energy.

\subsection{Nonwoven/rubber composites}

Results from previous section showed that sound absorption coefficients of the obtained nonwovens were high in a range of mid-high frequency $(2000-6000 \mathrm{~Hz})$. However, they were poor in a low-mid frequency range (100-2000 Hz). In order to improve sound absorption performance in such range, further investigation was made by inserting a $1 \mathrm{~mm}$-thick rubber sheet layer between the nonwoven layer and its sound absorption coefficients was measured, in comparison with the 2-layer nonwoven counterpart. In this study, the nonwovens of $7-4 \mathrm{H}$ and $15-\mathrm{H}$ fibers, which exhibited the highest and the lowest sound absorption performances, respectively, were employed. The perforated rubber sheets, having holes of 3-mm diameter and distance between holes of either $8 \mathrm{~mm}$ (RH8) or $16 \mathrm{~mm}$ (RH16), were employed in comparison with rubber sheet without perforation (R) (Fig. 2). Figure 5a showed that the $15-\mathrm{H}$ nonwovens with inserted rubber layer $(15-\mathrm{H} / \mathrm{R} / 15-$ $\mathrm{H})$ exhibited significant increase in sound absorption coefficient at a low frequency range. It showed a maximum value, $a_{\max }=0.92$ at $630 \mathrm{~Hz}$ before dropping over a frequency range of $800-2200 \mathrm{~Hz}$ and then increased to reach a plateau. The drop in sound absorption coefficient value could be resulted from the middle rubber layer that blocked sound wave from reaching the next nonwoven layer. The latter nonwoven layer, thus, could not fully contribute to sound absorption performance. Similar trend was observed in the 7-4H/R/7-4H nonwoven (Fig. 5b). For further study, the perforated rubber sheet (either $\mathrm{RH} 8$ or $\mathrm{RH} 16$ ) was inserted between nonwoven layers and their sound absorption coefficients were measured. As shown in Fig. 5a (dotted line), the 15-H/RH16/15-H composite exhibited a sound absorption peak $\left(a_{\max }=0.90\right)$ at a higher frequency $(1000 \mathrm{~Hz})$, compared to that of no perforation $(15-\mathrm{H} / \mathrm{R} / 15-\mathrm{H})$. For the $15-\mathrm{H} / \mathrm{RH} 8 / 15-\mathrm{H}$ composite with higher percent of perforation (11\%), the sound absorption peak $\left(a_{\max }=0.89\right)$ shifted towards higher frequency $(2000 \mathrm{~Hz})$ as shown in Fig. 5a (dashed line). Furthermore, a drop 


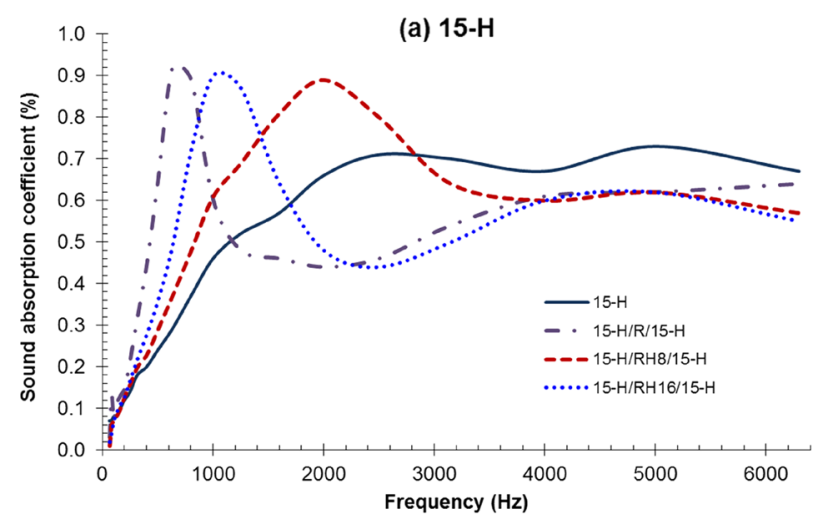

(b) $7-4 \mathrm{H}$

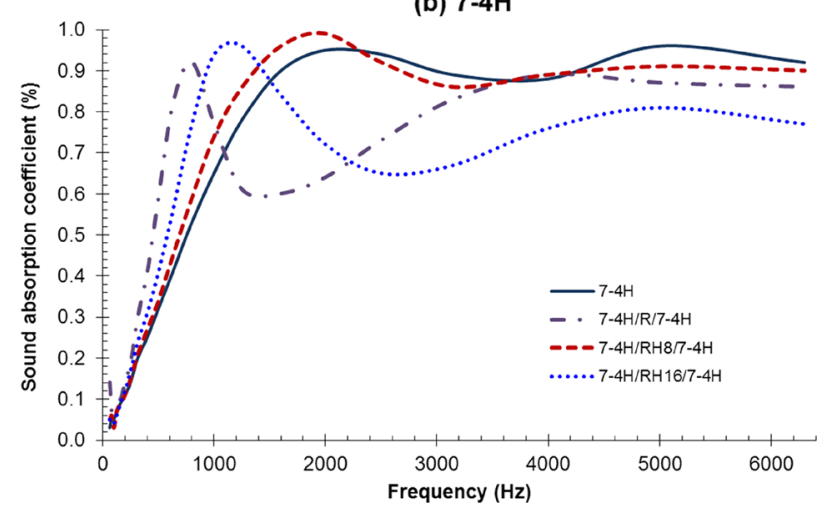

Fig. 5 Sound absorption coefficients of nonwoven-rubber composites. a $15-\mathrm{H}$ and $\mathbf{b}$ 7-4 $\mathrm{H}$ fibers; Rubber with and without perforation (RH8, RH16, and R)

in sound absorption coefficient afterwards became less, compared to that of $15-\mathrm{H} / \mathrm{RH} 16 / 15-\mathrm{H}$ (perforation $=3 \%$ ). Similar observations were made in the $7-4 \mathrm{H}$ / R16/7-4H and 7-4H/R8/7-4H composites, where peaks of sound absorption coefficients $\left(a_{\max }=0.96\right.$ and 0.99 , respectively) shifted towards higher frequency ( 1250 and $2000 \mathrm{~Hz}$, respectively) as percent of perforation in the rubber layer increased (Fig. 5b). This observation was in good agreement with that reported by Lou et al. [16] where absorption peak shifted towards higher frequency with increasing percent of perforation in the flexible PU-foam/nonwoven composites. In our study, the perforated rubber sheet retarded sound wave propagation via sound interference between the neighboring holes. In addition, it allowed sound waves to propagate through holes and reached the latter nonwoven layer. Thus, sound absorption contribution from the nonwoven layer could be obtained. From these results, it was demonstrated that addition of perforated rubber layer to nonwoven could improve sound absorption coefficients, especially in a low frequency range.

\section{Conclusions}

A comprehensive study on sound absorption properties of needle-punched polyester nonwovens was conducted, where polyester fibers of different sizes ( 7 and 15 denier) and configurations (hollow, 4-hole, hollow conjugated, and hollow trilobal) were employed to investigate their effects on sound absorption performance of the nonwovens. Sound absorption coefficient was found to increase with increasing number of nonwoven layers (from 1 to 2 and 3 layers) due to an increase in thickness and presence of air gap between the layers. Nonwovens with finer fibers (7 denier) possessed longer tortuous paths and larger surface areas, compared to those with coarser fibers (15 denier), resulting in their higher sound absorption coefficients. For fiber configurations, nonwovens of 4-hole fibers $(7-4 \mathrm{H})$ showed a higher sound absorption coefficient than that of single-hole fibers $(7-\mathrm{H})$ due to larger surface areas of small holes in the fibers. Larger hollow area in the hollow conjugated fibers $(15-\mathrm{HC})$ had a greater sound absorption coefficient, compared to those of hollow fiber counterpart (15-H). Results from this study suggested that nonwovens of fine fibers with irregular shapes and large hole area should be employed for obtaining good sound absorption properties. In addition, it was demonstrated that insertion of perforated elastic rubber layer could enhance the sound absorption performance at a low-frequency range of the nonwoven-rubber composite. Knowledge obtained from this study enables design and fabrication of nonwoven composites with enhanced sound absorption performance over a broad frequency range. It is of our interest to extend research work on developing flexible nonwoven composite with high sound absorption performance.

Acknowledgements This work was supported by the National Metal and Materials Technology Center, Thailand National Science and Technology Development Agency.

\section{Compliance with ethical standards}

Conflict of interest The authors declare that they have no potential conflict of interest to this work.

\section{References}

1. Tang X, Yan X (2017) Acoustic energy absorption properties of fibrous materials: a review. Composites A 101:360-380

2. Sengupta $S$ (2010) Sound reduction by needle-punched nonwoven fabrics. Indian J Fibre Text Res 35:237-242 
3. Zhu W, Nandikolla V, George B (2015) Effect of bulk density on the acoustic performance of thermally bonded nonwovens. J Eng Fiber Fabr 10:39-45

4. Tascan M, Vaughn EA (2008) Effects of total surface area and fabric density on the acoustical behavior of needlepunched nonwoven fabrics. J Text I 78(4):289-296

5. Benkreira H, Khan A, Horoshenkov KV (2011) Sustainable acoustic and thermal insulation materials from elastomeric waste residues. Chem Eng Sci 66:4157-4171

6. Tang X, Yan X (2017) Multi-layer fibrous structures for noise reduction. J Text Inst 108(12):2096-2106

7. Abdelfattah AM, Ibrahim GE, Mahmoud ER (2011) Using nonwoven hollow fibers to improve cars interior acoustic properties. Life Sci J 8(1):344-351

8. Lee Y, Joo C (2003) Sound absorption properties of recycled polyester fibrous assembly absorbers. AUTEX Res J 3:72-77

9. Kino N, Ueno T (2008) Evaluation of acoustical and nonacoustical properties of sound absorbing materials made of polyester fibres of various cross-sectional shapes. Appl Acoust 69(7):575-582

10. Liu Z, Zhan J, Fard M, Davy JL (2017) Acoustic properties of multilayer sound absorbers with a 3D printed micro-perforated panel. Appl Acoust 121:25-32

11. Tayong R, Dupont $T$, Leclaire $P$ (2016) Experimental investigation of holes interaction effect on the sound absorption coefficient of micro-perforated panels under high and medium sound levels. Appl Acoust 72:777-784

12. Li D, Chang D, Liu B, Tian J (2014) Improving sound absorption bandwidth of micro-perforated panel by adding porous materials. In: Proceedings of inter noise, Melbourne, Australia, pp 1-6

13. Li D, Chang D, Liu B (2017) Enhanced low- to mid-frequency sound absorption using parallel-arranged perforated plates with extended tubes and porous material. Appl Acoust 127:316-323

14. Zhou H, Bo L, Guangsu H, Jia H (2007) A novel composite sound absorber with recycled rubber particles. J Sound Vib 304:400-406

15. Han Z, Chunsheng L, Kombe T, Thong-On N (2008) Crumb rubber blends in noise absorption study. Mater Struct 41:383-390

16. Lou CW, Huang SY, Huang CH, Pan YJ, Yan R, Hsieh CT, Lin JH (2015) Effects of structure design on resilience and acoustic absorption properties of porous flexible-foam based perforated composites. Fiber Polym 16(12):2652-2662

Publisher's Note Springer Nature remains neutral with regard to jurisdictional claims in published maps and institutional affiliations. 